\title{
Mean square Hyers-Ulam stability of stochastic differential equations driven by Brownian motion
}

\section{Xiangkui Zhao*}

\section{${ }^{*}$ Correspondence: \\ xiangkuizh@ustb.edu.cn \\ School of Mathematics and Physics, \\ University of Science and \\ Technology Beijing, Beijing, 100083, \\ China}

\begin{abstract}
In this paper, the Hyers-Ulam stability for a class of first order stochastic differential equations is studied by using the Ito formula. Furthermore, the research results are applied to a class of second order stochastic differential equations with constant coefficients by the substitution method. In the end, the Hyers-Ulam stability of general second order stochastic differential equations is considered by the solutions of two deterministic second order differential equation boundary value problems.
\end{abstract}

Keywords: Hyers-Ulam stability; stochastic differential equations; Brownian motion; substitution method

\section{Introduction}

The Hyers-Ulam stability of functional equations was introduced with the motivation of studying the stability of approximate solutions $[1,2]$. Since then, much attention was given to the stability studies of functional equations; see [3-6] and the references therein. In 1993, Obloza introduced the notion of Hyers-Ulam stability for the studies of differential equations $[7,8]$. Furthermore, the stability studies of differential equations have been considered in the recent decade; see [9-20] and the references therein. To the best of the author's knowledge, after the success of the investigations of the Hyers-Ulam stability for deterministic differential equations, there are a few arguments about the Hyers-Ulam stability of stochastic differential equations in the literature. However, uncertainty is involved in all kinds of natural phenomena, and stochastic differential equations are the suitable mathematical models for the natural phenomena. Therefore, it is important to generalize the research results of deterministic differential equations to stochastic differential equations. In the paper, we will consider the Hyers-Ulam stability of the following stochastic differential equations in the mean square which are perturbed by the Brownian motion:

$$
d X_{t}=\left(a_{t} X_{t}+f_{t}\right) d t+h_{t} d B_{t}
$$

and

$$
d X_{t}^{\prime}=\left(b_{t} X_{t}^{\prime}+c_{t} X_{t}+r_{t}\right) d t+k_{t} d B_{t}
$$

(c) 2016 Zhao. This article is distributed under the terms of the Creative Commons Attribution 4.0 International License (http://creativecommons.org/licenses/by/4.0/), which permits unrestricted use, distribution, and reproduction in any medium, provided you give appropriate credit to the original author(s) and the source, provide a link to the Creative Commons license, and indicate if changes were made. 
where $t \geq 0, a, b, c, f, h, r, k:[0,+\infty) \rightarrow \mathbb{R}$ are continuous, $B_{t}$ is a standard one-dimensional Brownian motion, $X_{t}$ is a stochastic process which is adapted to the same filtration as $B_{t}$. If $h_{t} \equiv 0, k_{t} \equiv 0$, equations (1.1) and (1.2) are deterministic equations, which had been considered by the method of integral factors in [13-15].

\section{Preliminary}

Now we introduce the fundamental definitions and a lemma, which are used later in the article. Throughout this paper, we consider a filtered probability space $(\Omega, \mathcal{F}, P)$ with filtration $\mathcal{F}_{t}, t \geq 0$ satisfying the usual conditions, that is, it is right continuous and increasing, while $\mathcal{F}_{0}$ contains all $P$-null sets.

Definition 2.1 Assume that for any $\varepsilon \geq 0$ and any stochastic process

$$
Y_{t} \in \mathcal{L}_{2}\left(\Omega, \mathcal{F},\left(\mathcal{F}_{t}\right), P\right)
$$

satisfies

$$
E\left(Y_{t}-\int_{0}^{t}\left(a Y_{s}+f_{s}\right) d s-\int_{0}^{t} h_{s} d B_{s}\right)^{2}<\varepsilon, \quad t \in(0, T)
$$

where $E$ is the expectation operator, then there exists a solution $X_{t}$ of equation (1.1) such that $\left|Y_{t}-X_{t}\right| \leq K \varepsilon, t \in(0, T)$ with $K$ is a positive real constant. We say that equation (1.1) is Hyers-Ulam stable on $(0, T)$ in the mean square.

Definition 2.2 Assume that for any $\varepsilon>0$ and any stochastic process

$$
Y_{t} \in \mathcal{L}_{2}\left(\Omega, \mathcal{F},\left(\mathcal{F}_{t}\right), P\right)
$$

satisfies the following inequality:

$$
E\left(Y_{t}^{\prime}-\int_{0}^{t}\left(b_{s} Y_{s}^{\prime}+c_{s} Y_{s}+r_{s}\right) d s-\int_{0}^{t} k_{s} d B_{s}\right)^{2}<\varepsilon, \quad t \in(0, T)
$$

where $E$ is the expectation operator, then there exists a solution $X_{t}$ of equation (1.2) such that $\left|Y_{t}-X_{t}\right| \leq K \varepsilon, t \in(0, T)$ with $K$ a positive real constant. We say that equation (1.2) is Hyers-Ulam stable on $(0, T)$ in the mean square.

To consider the integration of the stochastic process, we use the Ito formula as follows.

Lemma 2.1 ([21]) Suppose $d X_{t}=U_{t} d t+V_{t} d B_{t}$, where the vector $U=\left(U_{1}, \ldots, U_{m}\right)$ and the matrix $V=\left(V_{1}, \ldots, V_{m}\right)$ have $\mathcal{L}_{2}$ components and $B$ is the vector of $m$ independent Brownian motions. Let $F$ be a twice continuously differentiable function from $\mathbb{R}^{m}$ into $\mathbb{R}$. Then $Y_{t}=F\left(X_{t}\right)$ is also an Ito process and

$$
d Y_{t}=\sum_{i=1}^{m} \frac{\partial F}{\partial x_{i}}\left(X_{t}\right) d X_{i, t}+\frac{1}{2} \sum_{i, j=1}^{m} \frac{\partial^{2} F}{\partial x_{i} x_{j}}\left(X_{t}\right) d X_{i, t} \cdot d X_{j, t}
$$

where $d X_{i, t} \cdot d X_{j, t}$ is computed by using the rules $d t d t=d t d B_{i, t}=d B_{i, t} d t=0, d B_{i, t} d B_{j, t}=0$ for $i \neq j$ and $\left(d B_{i, t}\right)^{2}=d t$. 
Let $m=2, F\left(X_{t}\right)=X_{1, t} X_{2, t}$, then from Lemma 2.1, we see

$$
d F\left(X_{t}\right)=d X_{1, t} X_{2, t}=X_{2, t} d X_{1, t}+X_{1, t} d X_{2, t}+d X_{1, t} d X_{2, t}
$$

and

$$
X_{1, t} X_{2, t}=X_{1,0} X_{2,0}+\int_{0}^{t}\left(X_{2, s} d X_{1, s}+X_{1, s} d X_{2, s}+d X_{1, s} d X_{2, s}\right)
$$

\section{Hyers-Ulam stability of (1.1)}

In this section, we establish some criteria of the Hyers-Ulam stability of equation (1.1), by using the Ito formula.

Theorem 3.1 Let $Y_{t}$ be an Ito process, $a, f, h \in \mathcal{L}^{2}[0, T]$,

$$
d g\left(t, Y_{t}\right)=d Y_{t}-\left(a_{t} Y_{t}+f_{t}\right) d t-h_{t} d B_{t}
$$

assume that $Y_{t}$ satisfies $E\left(g\left(t, Y_{t}\right)\right)^{2} \leq \varepsilon$, for $t \in(0, T), \varepsilon \geq 0$. Then there exists a solution $X_{t}$ of equation (1.1) such that $X_{0}=Y_{0}, E\left(X_{t}-Y_{t}\right)^{2} \leq M \varepsilon$ with

$$
M_{1}=4 \max _{0 \leq t \leq T}\left(1+e^{\int_{0}^{t} a_{s} d s}\right)^{2} .
$$

That means equation (1.1) is Hyers-Ulam stable in the mean square on the interval $(0, T)$.

Proof Multiplying two sides of (3.1) by the function $e^{-\int_{0}^{t} a_{s} d s}$, we obtain

$$
e^{-\int_{0}^{t} a_{s} d s}\left(d g\left(t, Y_{t}\right)+f_{t} d t+h_{t} d B_{t}\right)=e^{-\int_{0}^{t} a_{s} d s}\left(d Y_{t}-a Y_{t} d t\right) .
$$

Applying Lemma 2.1, we have

$$
\begin{aligned}
d\left(e^{-\int_{0}^{t} a_{s} d s} Y_{t}\right) & =Y_{t} d e^{-\int_{0}^{t} a_{s} d s}+e^{-\int_{0}^{t} a_{s} d s} d Y_{t}+d e^{-\int_{0}^{t} a_{s} d s} d Y_{t} \\
& =e^{-\int_{0}^{t} a_{s} d s}\left(d Y_{t}-a_{t} Y_{t} d t\right) .
\end{aligned}
$$

From (3.2), we have

$$
e^{-\int_{0}^{t} a_{s} d s}\left(d g\left(t, Y_{t}\right)+f_{t} d t+h_{t} d B_{t}\right)=e^{-\int_{0}^{t} a_{s} d s}\left(d Y_{t}-a_{t} Y_{t} d t\right)=d\left(e^{-\int_{0}^{t} a_{s} d s} Y_{t}\right) .
$$

Integrating the two sides of (3.3) from 0 to $t$ and multiplying the two sides of (3.3) by the function $e^{\int_{0}^{t} a_{s} d s}$, we get

$$
e^{\int_{0}^{t} a_{s} d s} Y_{0}+e^{\int_{0}^{t} a_{s} d s} \int_{0}^{t} e^{-\int_{0}^{s} a_{\tau} d \tau}\left(f_{s} d s+h_{s} d B_{s}\right)+e^{\int_{0}^{t} a_{s} d s} \int_{0}^{t} e^{-\int_{0}^{s} a_{\tau} d \tau} d g\left(s, Y_{s}\right)=Y_{t} .
$$

Define

$$
X_{t}:=e^{\int_{0}^{t} a_{s} d s} Y_{0}+e^{\int_{0}^{t} a_{s} d s} \int_{0}^{t} e^{-\int_{0}^{s} a_{\tau} d \tau}\left(f_{s} d s+h_{s} d B_{s}\right)
$$


then we have $X_{0}=Y_{0}$ and

$$
\begin{aligned}
d X_{t}= & Y_{0} a_{t} e^{\int_{0}^{t} a_{s} d s} d t+\left(\int_{0}^{t} e^{-\int_{0}^{s} a_{\tau} d \tau}\left(f_{s} d s+h_{s} d B_{s}\right)\right) d e^{\int_{0}^{t} a_{s} d s} \\
& +e^{\int_{0}^{t} a_{s} d s} d \int_{0}^{t} e^{-\int_{0}^{s} a_{\tau} d \tau}\left(f_{s} d s+h_{s} d B_{s}\right) \\
& +d e^{\int_{0}^{t} a_{s} d s} d \int_{0}^{t} e^{-\int_{0}^{s} a_{\tau} d \tau}\left(f_{s} d s+h_{s} d B_{s}\right) \\
= & \left(a_{t} X_{t}+f_{t}\right) d t+h_{t} d B_{t} .
\end{aligned}
$$

Hence $X_{t}$ is a solution of equation (1.1). We rewrite (3.4) as

$$
X_{t}-Y_{t}=-e^{\int_{0}^{t} a_{s} d s} \int_{0}^{t} e^{-\int_{0}^{s} a_{\tau} d \tau} d g\left(s, Y_{s}\right)
$$

Applying Lemma 2.1, we have

$$
\int_{0}^{t} e^{-\int_{0}^{s} a_{\tau} d \tau} d g\left(s, Y_{s}\right)=e^{-\int_{0}^{t} a_{s} d s} g\left(t, Y_{t}\right)-g\left(0, Y_{0}\right)+\int_{0}^{t} g\left(s, Y_{s}\right) d e^{-\int_{0}^{s} a_{\tau} d \tau},
$$

where $\int_{0}^{t} g\left(s, Y_{s}\right) d e^{-\int_{0}^{s} a_{\tau} d \tau}$ is a Stieltjes integral. Taking expectations on the two sides of (3.5), we see

$$
E\left(X_{t}-Y_{t}\right)^{2} \leq\left(1+e^{\int_{0}^{t} a_{s} d s}+\left|e^{\int_{0}^{t} a_{s} d s} \int_{0}^{t} a_{s} e^{-\int_{0}^{s} a_{\tau} d \tau} d s\right|\right)^{2} \varepsilon \leq 4\left(1+e^{\int_{0}^{t} a_{s} d s}\right)^{2} \varepsilon \leq M_{1} \varepsilon
$$

on the interval $[0, T]$ by (3.6). Hence equation (1.1) is Hyers-Ulam stable in the mean square on the interval $[0, T]$. The proof is completed.

\section{Hyers-Ulam stability of (1.2)}

First of all, we consider the Hyers-Ulam stability of equation (1.2) by using the substitution method for a special case. We assume that $b_{t}$ and $c_{t}$ are both constant functions and write $b$ and $c$ instead of $b_{t}$ and $c_{t}$.

Theorem 4.1 Let $Y_{t}^{\prime}$ be an Ito process,

$$
d G\left(t, Y_{t}\right)=d Y_{t}^{\prime}-\left(b Y_{t}^{\prime}+c Y_{t}+r_{t}\right) d t-k_{t} d B_{t}
$$

Assume that $E\left(G\left(t, Y_{t}\right)\right)^{2} \leq \varepsilon$ for $t \in(0, T), \varepsilon \geq 0$. Then there exists a solution $X_{t}$ of equation (1.2) such that

$$
E\left(X_{t}-Y_{t}\right)^{2} \leq M_{2} \varepsilon, \quad t \in(0, T),
$$

with

$$
\begin{aligned}
& X_{0}=Y_{0}, \quad X_{0}^{\prime}=Y_{0}^{\prime}, \\
& M_{2}=\left(1+(1+|b|) \theta+|c|(1+|b|) T \theta^{2}+(1+|b|)\left(b^{2}+c+|b|\right) T \theta^{2}\right)^{2},
\end{aligned}
$$




$$
\begin{aligned}
& \theta=\frac{e^{\frac{T\left(|b|+\sqrt{\left.b^{2}+4 c\right)}\right.}{2}}}{\sqrt{b^{2}+4 c}} \max \left\{1,|b|+\sqrt{b^{2}+4 c}\right\} \quad \text { when } b^{2}+4 c>0 ; \\
& \theta=\frac{2 e^{\frac{T|b|}{2}}}{\sqrt{b^{2}+4 c}} \max \{1, \sqrt{-c}\} \quad \text { when } b^{2}+4 c<0 ; \\
& \theta=e^{\frac{T|b|}{2}} \max \left\{\frac{|b|}{2},\left|1-\frac{b^{2}}{4}\right|\right\} \quad \text { when } b^{2}+4 c=0 .
\end{aligned}
$$

That means equation (1.2) is Hyers-Ulam stable in the mean square on the interval [0,T].

Proof Let

$$
Z_{t}=\left(\begin{array}{c}
X_{t} \\
\dot{X}_{t}
\end{array}\right), \quad U_{t}=\left(\begin{array}{c}
Y_{t} \\
\dot{Y}_{t}
\end{array}\right), \quad A=\left(\begin{array}{cc}
0 & 1 \\
c & b
\end{array}\right), \quad B=\left(\begin{array}{l}
0 \\
1
\end{array}\right),
$$

then (1.2) can be rewritten as

$$
d Z_{t}=\left(A Z_{t}+B r_{t}\right) d t+B k_{t} d B_{t}
$$

We write

$$
B d G\left(t, Y_{t}\right)=d U_{t}-\left(A U_{t}+B r_{t}\right) d t-B k_{t} d B_{t}
$$

instead of (4.1). Multiplying two sides of (4.3) by the matrix function $e^{-A t}$, we get

$$
e^{-A t} B d G\left(t, Y_{t}\right)=e^{-A t}\left(d U_{t}-A U_{t}\right)-e^{-A t}\left(B r_{t} d t+B k_{t} d B_{t}\right)
$$

Since $Y_{t}^{\prime}$ is an Ito process, without loss of generality, we can define

$$
d Y_{t}^{\prime}:=U_{t}^{\dot{y}} d t+V_{t}^{\dot{y}} d B_{t}
$$

By computing, we have

$$
d Y_{t}=\left(\int_{0}^{t} U_{s}^{\dot{y}} d s\right) d t+\left(\int_{0}^{t} V_{s}^{\dot{y}} d B_{s}\right) d t
$$

By Lemma 2.1, we see $d t d U_{t}=d t\left(d Y_{t}^{\prime}, d Y_{t}\right)=0$. Hence

$$
\begin{aligned}
d e^{-A t} U_{t} & =\left(d e^{-A t}\right) U_{t}+e^{-A t}\left(d U_{t}\right)+d e^{-A t} d U_{t} \\
& =e^{-A t}\left(d U_{t}-A U_{t} d t\right)
\end{aligned}
$$

From (4.4), we have

$$
e^{-A t} B d G\left(t, Y_{t}\right)=d e^{-A t} U_{t}-e^{-A t}\left(B r_{t} d t+B k_{t} d B_{t}\right)
$$

Integrating two sides of (4.5) from 0 to $t$ and multiplying (4.5) by the matrix function $e^{A t}$, we see

$$
e^{A t} U_{0}+e^{A t} \int_{0}^{t} e^{-A s}\left(B r_{s} d s+B k_{s} d B_{s}\right)=U_{t}-e^{A t} \int_{0}^{t} e^{-A s} B d G\left(s, Y_{s}\right)
$$


Define

$$
Z_{t}:=e^{A t} U_{0}+e^{A t} \int_{0}^{t} e^{-A s}\left(B r_{s} d s+B k_{s} d B_{s}\right)
$$

Then we have

$$
\begin{aligned}
d Z_{t}= & A e^{A t} U_{0} d t+B r_{t} d t+B k_{t} d B_{t}+A e^{A t} d t \int_{0}^{t} e^{-A s}\left(B r_{s} d s+B k_{s} d B_{s}\right) \\
& +d e^{A t} d \int_{0}^{t} e^{-A s}\left(B r_{s} d s+B k_{s} d B_{s}\right) \\
= & \left(A Z_{t}+B r_{t}\right) d t+B k_{t} d B_{t} .
\end{aligned}
$$

Therefore $Z_{t}=\left(X_{t}, X_{t}^{\prime}\right)^{T}$ is a solution of (4.2), that is, $X_{t}$ is a solution of (1.2) with $X_{0}=Y_{0}$, $X_{0}^{\prime}=Y_{0}^{\prime}$. We rewrite (4.6) as

$$
Z_{t}-U_{t}=-e^{A t} \int_{0}^{t} e^{-A s} B d G\left(s, Y_{s}\right)
$$

Similar to Theorem 3.1, by Lemma 2.1, we have

$$
\int_{0}^{t} e^{-A s} B d G\left(s, Y_{s}\right)=e^{-A t} B G\left(t, Y_{t}\right)-B G\left(0, Y_{0}\right)+\int_{0}^{t} A e^{-A s} B G\left(s, Y_{s}\right) d s .
$$

By (4.7) and (4.8), we have

$$
Z_{t}-U_{t}=-B G\left(t, U_{t}\right)+e^{A t} B G\left(0, Y_{0}\right)-e^{A t} \int_{0}^{t} A e^{-A s} B G\left(s, Y_{s}\right) d s
$$

Assume

$$
e^{A t}=\alpha(t) A+\beta(t) E
$$

with $E$ the identity matrix. Hence

$$
\begin{aligned}
& e^{A t}=\alpha(t) A+\beta(t) E=\left(\begin{array}{cc}
\beta(t) & \alpha(t) \\
\alpha(t) c & \alpha(t) b+\beta(t)
\end{array}\right), \\
& e^{A t} B=(\alpha(t) A+\beta(t) E) B=\left(\begin{array}{c}
\alpha(t) \\
\alpha(t) b+\beta(t)
\end{array}\right), \\
& \int_{0}^{t} A e^{-A s} B G\left(s, Y_{s}\right) d s=\left(\begin{array}{c}
\int_{0}^{t}(\alpha(-s) b+\beta(-s)) G\left(s, Y_{s}\right) d s \\
\int_{0}^{t}\left(\alpha(-s)\left(b^{2}+c\right)+\beta(-s) b\right) G\left(s, Y_{s}\right) d s
\end{array}\right) .
\end{aligned}
$$

By (4.8), (4.9), (4.10), (4.11), (4.12), (4.13), we have

$$
\begin{aligned}
\left(X_{t}-Y_{t}\right)^{2}= & \left(-G\left(t, Y_{t}\right)+(\alpha(t) b+\beta(t)) G\left(0, Y_{0}\right)+\alpha(t) c \int_{0}^{t}(\alpha(-s) b+\beta(-s)) G\left(s, Y_{s}\right) d s\right. \\
& \left.+(\alpha(t) b+\beta(t)) \int_{0}^{t}\left(\alpha(-s)\left(b^{2}+c\right)+\beta(-s) b\right) G\left(s, Y_{s}\right) d s\right)^{2}
\end{aligned}
$$


We consider three possibilities for computing $\alpha(t), \beta(t)$.

(i) If $b^{2}+4 c>0$, we see that

$$
\lambda_{1}=\frac{b+\sqrt{b^{2}+4 c}}{2}, \quad \lambda_{2}=\frac{b-\sqrt{b^{2}+4 c}}{2}
$$

are different real eigenvalues of the matrix $A$. By (4.10), we have

$$
\left\{\begin{array}{l}
e^{\lambda_{1} t}=\alpha(t) \lambda_{1}+\beta(t) \\
e^{\lambda_{2} t}=\alpha(t) \lambda_{2}+\beta(t) .
\end{array}\right.
$$

Hence

$$
\begin{aligned}
& \alpha(t)=\frac{e^{\frac{b}{2} t}\left(e^{\frac{t \sqrt{b^{2}+4 c}}{2}}-e^{-\frac{t \sqrt{b^{2}+4 c}}{2}}\right)}{\sqrt{b^{2}+4 c}}, \\
& \beta(t)=\frac{\left(\frac{b+\sqrt{b^{2}+4 c}}{2}\right) e^{\left.\frac{b-\sqrt{b^{2}+4 c}}{2}\right) t}-\left(\frac{b-\sqrt{b^{2}+4 c}}{2}\right) e^{\left(\frac{b+\sqrt{b^{2}+4 c}}{2}\right) t}}{\sqrt{b^{2}+4 c}} .
\end{aligned}
$$

(ii) If $b^{2}+4 c<0$, we see that

$$
\lambda_{1}=\frac{b+i \sqrt{\left|b^{2}+4 c\right|}}{2}, \quad \lambda_{2}=\frac{b-i \sqrt{\left|b^{2}+4 c\right|}}{2},
$$

are two different complex eigenvalues. By (4.10), we have

$$
\left\{\begin{array}{l}
e^{\lambda_{1} t}=e^{\frac{b}{2} t}\left(\cos \frac{t \sqrt{\left|b^{2}+4 c\right|}}{2}+i \sin \frac{t \sqrt{\left|b^{2}+4 c\right|}}{2}\right)=\alpha(t) \lambda_{1}+\beta(t), \\
e^{\lambda_{2} t}=e^{\frac{b}{2} t}\left(\cos \frac{t \sqrt{\left|b^{2}+4 c\right|}}{2}-i \sin \frac{t \sqrt{\left|b^{2}+4 c\right|}}{2}\right)=\alpha(t) \lambda_{2}+\beta(t) .
\end{array}\right.
$$

Hence

$$
\begin{aligned}
& \alpha(t)=\frac{2 e^{\frac{b}{2} t} \sin \left(\frac{t \sqrt{\left|b^{2}+4 c\right|}}{2}\right)}{\sqrt{\left|b^{2}+4 c\right|}}, \\
& \beta(t)=\frac{2 e^{\frac{b}{2} t}\left(\frac{\sqrt{\left|b^{2}+4 c\right|}}{2} \cos \frac{t \sqrt{\left|b^{2}+4 c\right|}}{2}-\frac{b}{2} \sin \frac{t \sqrt{\left|b^{2}+4 c\right|}}{2}\right)}{\sqrt{\left|b^{2}+4 c\right|}} .
\end{aligned}
$$

(iii) If $b^{2}+4 c=0$, we see that $\lambda_{1}=\lambda_{2}=\frac{b}{2}$. By (4.10), we have

$$
\left\{\begin{array}{l}
e^{\lambda_{1} t}=\alpha(t) \lambda_{1}+\beta(t) \\
\lambda_{1} e^{\lambda_{1} t}=\alpha(t)
\end{array}\right.
$$

Hence

$$
\alpha(t)=\frac{b}{2} e^{\frac{b}{2} t}, \quad \beta(t)=\left(1-\frac{b^{2}}{4}\right) e^{\frac{b}{2} t} .
$$

Taking expectations on the two sides of (4.14), we have

$$
E\left(X_{t}-Y_{t}\right)^{2} \leq\left(1+(1+|b|) \theta+|c|(1+|b|) T \theta^{2}+(1+|b|)\left(b^{2}+c+|b|\right) T \theta^{2}\right)^{2} \varepsilon=M_{2} \varepsilon
$$


with

$$
\begin{aligned}
& \theta=\frac{e^{\frac{T\left(|b|+\sqrt{b^{2}+4 c}\right)}{2}}}{\sqrt{b^{2}+4 c}} \max \left\{1,|b|+\sqrt{b^{2}+4 c}\right\} \quad \text { when } b^{2}+4 c>0 ; \\
& \theta=\frac{2 e^{\frac{T|b|}{2}}}{\sqrt{b^{2}+4 c}} \max \{1, \sqrt{-c}\} \quad \text { when } b^{2}+4 c>0 ; \\
& \theta=e^{\frac{T|b|}{2}} \max \left\{\frac{|b|}{2},\left|1-\frac{b^{2}}{4}\right|\right\} \quad \text { when } b^{2}+4 c=0 .
\end{aligned}
$$

Hence equation (1.2) is Hyers-Ulam stable in the mean square on the interval $[0, T]$. The proof is completed.

Since matrix multiplication is, in general, not commutative, Theorem 4.1 is not suitable for equation (1.2), when $b_{t}$ is not a constant function or $c_{t}$ is not a constant function. Now, we consider equation (1.2) by the solutions of two deterministic boundary value problems.

Let $u$ and $v$ be the solutions of the boundary value problems

$$
\left\{\begin{array}{l}
x_{t}^{\prime \prime}-b_{t} x_{t}^{\prime}-c_{t} x_{t}=0, \quad t \in(0, T), \\
u_{0}=0, \quad u_{T}=1,
\end{array}\right.
$$

and

$$
\left\{\begin{array}{l}
x_{t}^{\prime \prime}-b_{t} x_{t}^{\prime}-c_{t} x_{t}=0, \quad t \in(0, T), \\
u_{0}=1, \quad u_{T}=0,
\end{array}\right.
$$

respectively. Define

$$
\begin{aligned}
& p:=e^{-\int_{0}^{t} b_{s} d s,} \quad \rho:=u_{0}^{\prime}, \\
& \Lambda_{t, s}= \begin{cases}u_{s}^{\prime} v_{t}, & 0 \leq s \leq t \leq T, \\
u_{t} v_{s}^{\prime}, & 0 \leq t \leq s \leq T .\end{cases}
\end{aligned}
$$

Lemma 4.2 Let $X_{t}^{\prime}$ be an Ito process, $B_{t}$ is a standard one-dimensional Brownian motion. Assume that $p, b, c \in L^{2}[0, T]$, then

$$
X_{t}=\frac{1}{\rho} \int_{0}^{t}\left(\Lambda_{t, s} \int_{0}^{s} p_{\tau}\left(d X_{\tau}^{\prime}-\left(b_{\tau} X_{\tau}^{\prime}+c_{\tau} X_{\tau}\right) d \tau\right)\right) d s+v_{t} X_{0}+u_{t} X_{T}
$$

Proof Let

$$
Y_{s}=\int_{0}^{s} p_{\tau}\left(d X_{\tau}^{\prime}-\left(b_{\tau} X_{\tau}^{\prime}+c_{\tau} X_{\tau}\right) d \tau\right)
$$

By Lemma 2.1, we have

$$
Y_{s}=\int_{0}^{s}\left(p_{\tau} d X_{\tau}^{\prime}+X_{\tau}^{\prime} d p_{\tau}+d X_{\tau}^{\prime} d p_{\tau}\right)-\int_{0}^{s} p_{\tau} c_{\tau} X_{\tau} d \tau=p_{s} X_{s}^{\prime}-X_{0}^{\prime}-\int_{0}^{s} p_{\tau} c_{\tau} X_{\tau} d \tau
$$


Multiplying by the function $u_{s}^{\prime}$, integrating two sides of (4.18) from 0 to $t$, we see

$$
\begin{aligned}
\int_{0}^{t} u_{s}^{\prime} Y_{s} d s= & u_{t}^{\prime} p_{t} X_{t}-u_{0}^{\prime} X_{0}^{\prime}-\int_{0}^{t} p_{s}\left(u_{s}^{\prime \prime}-b_{s} u_{s}^{\prime}\right) X_{s} d s-\int_{0}^{t} d\left(u_{s}^{\prime} p_{s}\right) d X_{s}^{\prime}-u_{t} X_{0}^{\prime} \\
& -u_{t} \int_{0}^{t} p_{s} c_{s} X_{s} d s+\int_{0}^{t} u_{s} p_{s} c_{s} X_{s} d s+\int_{0}^{t} d u_{s}^{\prime} d \int_{0}^{s} p_{\tau} c_{\tau} X_{\tau} d \tau \\
= & u_{t}^{\prime} p_{t} X_{t}-u_{0}^{\prime} X_{0}-u_{t} X_{0}^{\prime}-u_{t} \int_{0}^{t} p_{s} c_{s} X_{s} d s
\end{aligned}
$$

Similarly, multiplying by the function $v_{s}^{\prime}$, integrating two sides of (4.18) from $t$ to $T$, we see

$$
\int_{t}^{T} v_{s}^{\prime} Y_{s} d s=-v_{t}^{\prime} p_{t} X_{t}+v_{T}^{\prime} p_{T} X_{T}+v_{t} X_{0}^{\prime}+v_{t} \int_{0}^{t} p_{s} c_{s} Y_{s} d s
$$

Therefore, by Abel's differential equation identity, we have

$$
\begin{aligned}
\frac{1}{\rho} \int_{0}^{T} \Lambda_{t, s} Y_{s} d s & =\frac{1}{u_{0}^{\prime}}\left(u_{t}^{\prime} v_{t}-u_{t} v_{t}^{\prime}\right) p_{t} X_{t}-\frac{1}{u_{0}^{\prime}}\left(v_{t} u_{0}^{\prime} X_{0}-u_{t} v_{T}^{\prime} p_{T} X_{T}\right) \\
& =-\frac{1}{u_{0}^{\prime}}\left|\begin{array}{ll}
u_{t} & v_{t} \\
u_{t}^{\prime} & v_{t}^{\prime}
\end{array}\right| p_{t} X_{t}-v_{t} X_{0}-u_{t} X_{T}=-\frac{1}{u_{0}^{\prime}}\left|\begin{array}{cc}
u_{0} & v_{0} \\
u_{0}^{\prime} & v_{0}^{\prime}
\end{array}\right| X_{t}-v_{t} X_{0}-u_{t} X_{T} \\
& =X_{t}-v_{t} X_{0}-u_{t} X_{T}
\end{aligned}
$$

That is,

$$
X_{t}=\frac{1}{\rho} \int_{0}^{T}\left(\Lambda_{t, s} \int_{0}^{s} p_{\tau}\left(d X_{\tau}^{\prime}-\left(b_{\tau} X_{\tau}^{\prime}+c_{\tau} X_{\tau}\right) d \tau\right)\right) d s+v_{t} X_{0}+u_{t} X_{T}
$$

The proof is completed.

Lemma 4.3 Let $B_{t}$ is a standard one-dimensional Brownian motion. $C, D$ are two stochastic variables. Assume that $p, r, k \in L^{2}[0, T]$, then the stochastic process

$$
X_{t}=\frac{1}{\rho} \int_{0}^{T}\left(\Lambda_{t, s} \int_{0}^{t} p_{s}\left(r_{s} d t+k_{s} d B_{s}\right)\right) d s+v_{t} C+u_{t} D, \quad 0<t<T
$$

is a solution of equation (1.2) such that $X_{0}=C, X(T)=D$.

Proof By Lemma 2.1, we obtain

$$
\begin{aligned}
& d \int_{0}^{t}\left(\Lambda_{t, s} \int_{0}^{t} p_{s}\left(r_{s} d t+k_{s} d B_{s}\right)\right) d s \\
& =v_{t}^{\prime} d t \int_{0}^{t}\left(u_{s}^{\prime} \int_{0}^{s} p_{\tau}\left(g_{\tau} d \tau+k_{\tau} d B_{\tau}\right)\right) d s+u_{t}^{\prime} v_{t}\left(\int_{0}^{t} p_{s}\left(r_{s} d \tau+k_{s} d B_{s}\right)\right) d t \\
& \quad+d v_{t} d \int_{0}^{t}\left(u_{s}^{\prime} \int_{0}^{s} p_{\tau}\left(g_{\tau} d \tau+k_{\tau} d B_{\tau}\right) d s\right) \\
& =v_{t}^{\prime} d t \int_{0}^{t}\left(u_{s}^{\prime} \int_{0}^{s} p_{\tau}\left(g_{\tau} d \tau+k_{\tau} d B_{\tau}\right)\right) d s+u_{t}^{\prime} v_{t}\left(\int_{0}^{t} p_{s}\left(r_{s} d s+k_{s} d B_{s}\right)\right) d t
\end{aligned}
$$




$$
\begin{aligned}
d \int_{0}^{t} & \Lambda_{t, s}\left(\int_{0}^{t} p_{s}\left(r_{s} d t+k_{s} d B_{s}\right) d s\right)^{\prime} \\
= & v_{t}^{\prime \prime} d t \int_{0}^{t}\left(u_{s}^{\prime} \int_{0}^{s} p_{\tau}\left(g_{\tau} d \tau+k_{\tau} d B_{\tau}\right)\right) d s+u_{t}^{\prime} v_{t}^{\prime}\left(\int_{0}^{t} p_{s}\left(r_{s} d \tau+k_{s} d B_{s}\right)\right) d t \\
& +d v_{t}^{\prime} d \int_{0}^{t}\left(u_{s}^{\prime} \int_{0}^{s} p_{\tau}\left(r_{\tau} d \tau+k_{\tau} d B_{\tau}\right)\right) d s+\left(u_{t}^{\prime} v_{t}^{\prime}+u_{t}^{\prime \prime} v_{t}\right) d t \int_{0}^{t} p_{s}\left(r_{s} d s+k_{s} d B_{s}\right) \\
& +u_{t}^{\prime} v_{t} p_{t}\left(\alpha(t) d t+k_{t} d B_{t}\right)+d\left(u_{t}^{\prime} v_{t}\right) d\left(\int_{0}^{t} p_{s}\left(r_{s} d s+k_{s} d B_{s}\right)\right) \\
= & v_{t}^{\prime \prime} d t \int_{0}^{t}\left(u_{s}^{\prime} \int_{0}^{s} p_{\tau}\left(r_{\tau} d \tau+k_{\tau} d B_{\tau}\right)\right) d s+u_{t}^{\prime} v_{t}^{\prime}\left(\int_{0}^{t} p_{s}\left(r_{s} d \tau+k_{s} d B_{s}\right)\right) d t \\
& +\left(u_{t}^{\prime} v_{t}^{\prime}+u_{t}^{\prime \prime} v_{t}\right) d t \int_{0}^{t} p_{s}\left(r_{s} d s+k_{s} d B_{s}\right)+u_{t}^{\prime} v_{t} p_{t}\left(\alpha(t) d t+h_{t} d B_{t}\right) .
\end{aligned}
$$

Similarly, we have

$$
\begin{aligned}
& d \int_{t}^{T}\left(\Lambda_{t, s} \int_{0}^{t} p_{s}\left(r_{s} d t+k_{s} d B_{s}\right)\right) d s \\
& =u_{t}^{\prime} d t \int_{t}^{T}\left(v_{s}^{\prime} \int_{0}^{s} p_{\tau}\left(r_{\tau} d \tau+k_{\tau} d B_{\tau}\right)\right) d s-u_{t} v_{t}^{\prime}\left(\int_{0}^{t} p_{s}\left(r_{s} d s+k_{s} d B_{s}\right)\right) d t \\
& d \int_{t}^{T} \Lambda_{t, s}\left(\int_{0}^{t} p_{s}\left(r_{s} d t+k_{s} d B_{s}\right) d s\right)^{\prime} \\
& =u_{t}^{\prime \prime} d t \int_{t}^{T}\left(v_{s}^{\prime} \int_{0}^{s} p_{\tau}\left(r_{\tau} d \tau+k_{\tau} d B_{\tau}\right)\right) d s-u_{t}^{\prime} v_{t}^{\prime}\left(\int_{0}^{t} p_{s}\left(r_{s} d \tau+k_{s} d B_{s}\right)\right) d t \\
& \quad-\left(u_{t}^{\prime} v_{t}^{\prime}+u_{t} v_{t}^{\prime \prime}\right) d t \int_{0}^{t} p_{s}\left(r_{\tau} d \tau+k_{\tau} d B_{\tau}\right)-u_{t} v_{t}^{\prime} p_{t}\left(\alpha(t) d t+k_{t} d B_{t}\right) .
\end{aligned}
$$

Hence, by Abel's differential equation identity, we have

$$
\begin{aligned}
& d X_{t}^{\prime}-\left(b_{t} X_{t}^{\prime}+c_{t} X_{t}\right) d t=\frac{1}{\rho}\left(u_{t}^{\prime} v_{t}-u_{t} v_{t}^{\prime}\right) p_{t}\left(r_{t} d t+k_{t} d B_{t}\right) \\
& +\frac{1}{\rho}\left(v_{t}\left(u_{t}^{\prime \prime}-b_{t} u_{t}^{\prime}\right)-u_{t}\left(v_{t}^{\prime \prime}-b_{t} v_{t}^{\prime}\right)\right) d t \int_{0}^{t} p_{s}\left(r_{\tau} d \tau+k_{\tau} d B_{\tau}\right) \\
& +C\left(u_{t}^{\prime \prime}-b_{t} u_{t}^{\prime}-c_{t} u_{t}^{\prime}\right) d t \\
& +D\left(v_{t}^{\prime \prime}-b_{t} v_{t}^{\prime}-c_{t} v_{t}^{\prime}\right) d t \\
& =-\frac{1}{\rho}\left|\begin{array}{ll}
u_{t} & v_{t} \\
u_{t}^{\prime} & v_{t}^{\prime}
\end{array}\right| p_{t}\left(r_{t} d t+k_{t} d B_{t}\right) \\
& +\frac{1}{\rho}\left(c_{t} u_{t} v_{t}-c_{t} u_{t} v_{t}\right) d t \int_{0}^{t} p_{s}\left(r_{\tau} d \tau+k_{\tau} d B_{\tau}\right) \\
& =-\frac{1}{\rho}\left|\begin{array}{ll}
u_{0} & v_{0} \\
u_{0}^{\prime} & v_{0}^{\prime}
\end{array}\right| e^{\int_{0}^{t} b_{s} d s} p_{t}\left(r_{t} d t+k_{t} d B_{t}\right) \\
& =r_{t} d t+k_{t} d B_{t} \text {. }
\end{aligned}
$$

Therefore (4.19) is a solution of equation (1.2). 
Theorem 4.4 Let $Y_{t}^{\prime}$ be an Ito process,

$$
d G\left(t, Y_{t}\right)=d Y_{t}^{\prime}-\left(b_{t} Y_{t}^{\prime}+c_{t} Y_{t}+r_{t}\right) d t-k_{t} d B_{t}
$$

Assume that $E\left(G\left(t, Y_{t}\right)\right)^{2} \leq \varepsilon$ for $t \in(0, T), \varepsilon \geq 0, b, c, r, k \in \mathcal{L}^{2}(0, T)$. Then there exists a solution $X_{t}$ of equation (1.2) such that

$$
E\left(X_{t}-Y_{t}\right)^{2} \leq M_{3} \varepsilon, \quad t \in(0, T)
$$

with

$$
\begin{aligned}
& X_{0}=Y_{0}, \quad X_{T}=Y_{T}, \\
& M_{3}=\frac{4}{\rho^{2}} \max _{t \in[0, T]}\left\{\left(\int_{0}^{T}\left|\Lambda_{t, s}\right|\left(p_{s}+1\right) d s\right)^{2}\right\} .
\end{aligned}
$$

That means equation (1.2) is Hyers-Ulam stable in the mean square on the interval $(0, T)$.

Proof By Lemma 4.2, we have

$$
Y_{t}=\frac{1}{\rho} \int_{0}^{T}\left(\Lambda_{t, s} \int_{0}^{s} p_{\tau}\left(d Y_{t}^{\prime}-\left(b_{t} Y_{t}^{\prime} d t+c_{t} Y_{t} d t\right)\right)\right) d s-v_{t} Y_{0}-u_{t} Y_{T}
$$

Let

$$
X_{t}=\frac{1}{\rho} \int_{0}^{T}\left(\Lambda_{t, s} \int_{0}^{s} p_{\tau}\left(r_{\tau} d \tau+k_{\tau} d B_{\tau}\right)\right) d s+v_{t} Y_{0}+u_{t} Y_{T},
$$

by Lemma 4.3, we obtain $X_{t}$ as a solution of equation (1.2) such that $X_{0}=Y_{0}, X_{T}=Y_{T}$. By (4.20), (4.21), (4.22), we get

$$
\frac{1}{\rho} \int_{0}^{T}\left(\Lambda_{t, s} \int_{0}^{s} p_{\tau} d G\left(\tau, Y_{\tau}\right)\right) d s=Y_{t}-X_{t} .
$$

By computing, we have

$$
\int_{0}^{s} p_{\tau} d G\left(\tau, Y_{\tau}\right)=p_{s} G\left(s, Y_{s}\right)-G\left(0, Y_{0}\right)-\int_{0}^{s} p_{\tau} b_{\tau} G\left(\tau, Y_{\tau}\right) d \tau .
$$

Taking expectations on the two sides of (4.23), we have

$$
\begin{aligned}
E\left(Y_{t}-Y_{t}\right)^{2} & =E\left(\frac{1}{\rho} \int_{0}^{T}\left(\Lambda_{t, s}\left(p_{s} G\left(s, Y_{s}\right)-G\left(0, Y_{0}\right)-\int_{0}^{s} p_{\tau} b_{\tau} G\left(\tau, Y_{\tau}\right) d \tau\right)\right) d s\right)^{2} \\
& \leq \frac{1}{\rho^{2}}\left(\int_{0}^{T}\left|\Lambda_{t, s}\right|\left(p_{s}+1+\left|\int_{0}^{s} p_{\tau} b_{\tau} d \tau\right|\right) d s\right)^{2} \varepsilon \\
& \leq \frac{4}{\rho^{2}}\left(\int_{0}^{T}\left|\Lambda_{t, s}\right|\left(p_{s}+1\right) d s\right)^{2} \varepsilon \\
& \leq M_{3} \varepsilon
\end{aligned}
$$

by (4.23). Hence equation (1.2) is Hyers-Ulam stable in the mean square on the interval $[0, T]$. The proof is completed. 


\section{Competing interests}

The author declares to have no conflict of interests regarding the publication of this paper.

\section{Acknowledgements}

Supported by the Fundamental Research Funds for the Central Universities.

Received: 3 June 2016 Accepted: 17 October 2016 Published online: 26 October 2016

\section{References}

1. Ulam, SM: A Collection of Mathematical Problems. Interscience, New York (1968)

2. Hyers, DH: On the stability of the linear functional equation. Proc. Natl. Acad. Sci. USA 27, $222-224$ (1941)

3. Zada, A, Shah, O, Shah, R: Hyers-Ulam stability of non-autonomous systems in terms of boundedness of Cauchy problems. Appl. Math. Comput. 271, 512-518 (2015)

4. Barbu, D, Buşe, C, Tabassum, A: Hyers-Ulam stability and discrete dichotomy. J. Math. Anal. Appl. 423, 1738-1752 (2015)

5. Lu, G, Park, C: Hyers-Ulam stability of additive set-valued functional equations. Appl. Math. Lett. 24, 1312-1316 (2011)

6. Zhang, D, Wang, J: On the Hyers-Ulam-Rassias stability of Jensen's equation. Bull. Korean Math. Soc. 46(4), 645-656 (2009)

7. Obloza, M: Hyers stability of the linear differential equation. Rocznik Nauk.-Dydakt. Prace Mat. 13, $259-270$ (1993)

8. Obloza, M: Connections between Hyers and Lyapunov stability of the ordinary differential equations. Rocznik Nauk.-Dydark. Prace Mat. 14, 141-146 (1997)

9. Abdollahpour, MR, Aghayari, R, Rassias, MTh: Hyers-Ulam stability of associated Laguerre differential equations in a subclass of analytic functions. J. Math. Anal. Appl. 437, 605-612 (2016)

10. Jung, SM: A fixed point approach to the stability of differential equations $y^{\prime}=F(x, y)$. Bull. Malays. Math. Soc. 33, 47-56 (2010)

11. Wang, J, Fečkan, M, Zhou, Y: Ulam's type stability of impulsive ordinary differential equations. J. Math. Anal. Appl. 395, 258-264 (2012)

12. Wang, J, LV, L, Zhou, Y: New concepts and results in stability of fractional differential equations. Commun. Nonlinear Sci. Numer. Simul. 17, 2530-2538 (2012)

13. Jung, SM: Hyers-Ulam stability of linear differential equations of first order, III. J. Math. Anal. Appl. 311, 139-146 (2005)

14. Li, Y, Shen, Y: Hyers-Ulam stability of linear differential equations of second order. Appl. Math. Lett. 23, 306-309 (2010)

15. Popa, D, Rasa, I: On the Hyers-Ulam stability of the linear differential equation. J. Math. Anal. Appl. 381, $530-537$ (2011)

16. András, S, Richárd Mészáros, A: Ulam-Hyers stability of dynamic equations on time scales via Picard operators. Appl. Math. Comput. 219, 4853-4864 (2013)

17. Diaz, JB, Margolis, B: A fixed point theorem of the alternative, for contractions on a generalized complete metric space. Bull. Am. Math. Soc. 74, 305-309 (1968)

18. Radu, V: The fixed point alternative and the stability of functional equations. Fixed Point Theory 4, 91-96 (2003)

19. Buşe, C, O'Regan, D, Saierli, O, Tabassum, A: Hyers-Ulam stability and discrete dichotomy for difference periodic systems. Bull. Sci. Math. 140, 908-934 (2016). doi:10.1016/j.bulsci.2016.03.010

20. Vinodkumar, A, Malar, K, Gowrisankar, M, Mohankumar, P: Existence, uniqueness and stability of random impulsive fractional differential equations. Acta Math. Sci. 36B(2), 428-442 (2016)

21. Øksendal, B: Stochastic Differential Equations: An Introduction with Applications. Springer, Heidelberg (1991)

\section{Submit your manuscript to a SpringerOpen ${ }^{\circ}$ journal and benefit from:}

- Convenient online submission

Rigorous peer review

- Immediate publication on acceptance

- Open access: articles freely available online

- High visibility within the field

- Retaining the copyright to your article 